\title{
TEMPOS MÁXIMOS DE FONAÇÃO E CARACTERÍSTICAS VOCAIS ACÚSTICAS DE MULHERES COM NÓDULOS VOCAIS
}

\author{
Maximum phonation time and vocal acoustic characteristics \\ for women with vocal fold nodule
}

\author{
Carla Aparecida Cielo ${ }^{(1)}$, Sabrina Schützenhofer Lasch (2), \\ Shanna Lara Miglioranzi ${ }^{(3)}$, Giseane Conterno ${ }^{(4)}$
}

\begin{abstract}
RESUMO
Objetivo: descrever os tempos máximos de fonação (TMF) e características vocais acústicas de mulheres adultas jovens com nódulos vocais. Método: coleta dos TMF das vogais /a/, /i/ e /u/; da relação s/z e medidas acústicas da fonte glótica (Multi Dimensional Voice Program Advanced da Kay PENTAX) de 20 sujeitos do sexo feminino, com faixa etária entre 20 e 40 anos, dez com laudo otorrinolaringológico de nódulos vocais e dez com laudo de laringe normal. Resultados: quanto aos TMF, o grupo sem nódulos (GSN) apresentou 90\% dos resultados normais e $10 \%$ diminuídos, e o grupo com nódulos (GCN) apresentou 70\% dos resultados normais e 30\% diminuídos; na relação s/z, todo o GSN apresentou resultados dentro dos padrões da normalidade e o GCN apresentou 70\% de resultados normais e $30 \%$ aumentados; ambos os grupos apresentaram todas as medidas vocais acústicas dentro da normalidade, com exceção do GSN que apresentou vf0 aumentada. Conclusão: as mulheres jovens com nódulos vocais apresentaram medidas de TMF e de relação s/z sem diferenças significativas em relação às mesmas medidas de mulheres sem afecção laríngea. As medidas acústicas da fonte glótica foram semelhantes e dentro da normalidade para ambos os grupos, com exceção da medida do cociente de variação de frequência fundamental (vf0) que foi elevada no GSN, sugerindo incoordenação penumofonoarticulatória. As medidas de TMF e da relação s/z, mesmo sem diferenças significativas, pareceram mais sensíveis à presença do predomínio aerodinâmico dos nódulos vocais do que as medidas acústicas.
\end{abstract}

DESCRITORES: Voz; Distúrbios da Voz; Cordas Vocais; Avaliação em Saúde

\section{INTRODUÇÃO}

Dá-se o nome de disfonia ao quadro caracterizado pela falta de harmonia vocal, impedindo a

(1) Fonoaudióloga; Professora Adjunta dos cursos de Graduação em Fonoaudiologia e Pós-Graduação em Distúrbios da Comunicação Humana do Departamento de Fonoaudiologia da Universidade Federal de Santa Maria, UFSM, Santa Maria, RS; Doutora em Linguística Aplicada pela Pontifícia Universidade Católica do Rio Grande do Sul.

(2) Fonoaudióloga; Policlínica Sarandi, Sarandi, RS; Mestre em Distúrbios da Comunicação Humana pela Universidade Federal de Santa Maria.

(3) Fonoaudióloga; Consultório particular, Santa Maria, RS; Mestranda em Distúrbios da Comunicação Humana pela Universidade Federal de Santa Maria.

(4) Fonoaudióloga; Consultório particular, Santa Maria, RS; Mestre em Distúrbios da Comunicação Humana pela Universidade Federal de Santa Maria.

Conflito de interesses: inexistente produção natural da voz bem como alterando a qualidade da emissão vocal ${ }^{1}$.

Os nódulos vocais são considerados disfonias organofuncionais ${ }^{1}$ de grande incidência ${ }^{2}$, formados por pequenas lesões laríngeas, com localização bilateral, benignas, de cor esbranquiçada, 1,3,4; observando-se edema e aumento de fibras colágenas na camada superficial da lâmina própria ${ }^{3,4}$. Situam-se classicamente na borda livre das pregas vocais ${ }^{2}$, na região anterior da glote e decorrem essencialmente de resposta ao abuso vocal ${ }^{1}$; ou a esforço vocal de intensidade e duração prolongadas, que resultam em tensão muscular, em sequência a processos inflamatórios laríngeos ${ }^{2}$.

Observa-se, ainda, o fechamento glótico incompleto, especialmente na área circunscrita ao nódulo; e disfonia de graus variados, dependendo do 
tamanho da lesão ${ }^{3,4}$, com presença principalmente de rouquidão e soprosidade ${ }^{5,6}$.

Em geral, em casos de nódulos vocais, a literatura aponta redução dos tempos máximos de fonação (TMF) ${ }^{1,7,8}$ e aumento da relação $s / \mathrm{z}^{1,7,8}$. Um estudo ${ }^{8}$ observou TMF de vogais e fricativas reduzidos em mulheres portadoras de nódulos vocais, no entanto apenas os TMF de vogais apresentaram diferença significativa. Em pesquisa ${ }^{9}$ que comparou um grupo de 40 professores com sintomas vocais freqüentes ou permanentes, sendo 30 deles com nódulos vocais, com um grupo controle de 40 professores com sintomas ausentes ou esporádicos, sendo 16 deles com nódulos vocais, os valores de relação $\mathrm{s} / \mathrm{z}$ encontraram-se dentro da normalidade em ambos os grupos.

As mesmas inconsistências de resultados ocorrem com os valores das medidas da avaliação acústica da voz. Estudos sugerem que, na presença de nódulos vocais, ocorre aumento das medidas de perturbação de frequência (jitter) ${ }^{1,2}$ e de amplitude (shimmer) 1,2,10, e das medidas de presença de ruído no sinal glótico ${ }^{2}$. Uma mesma pesquisa ${ }^{10}$ observou valores de shimmer aumentados num grupo de sujeitos com nódulos vocais e, no entanto, encontrou medidas de jitter similares entre o grupo com nódulos e o de controle. Além disso, diferentes programas de análise podem interferir nos valores destes parâmetros acústicos ${ }^{11,12}$.

Em função da falta de consenso na literatura sobre os resultados de medidas vocais acústicas e de TMF em casos de nódulos vocais e visando colaborar com os estudos voltados aos aspectos vocais de patologias laríngeas, justifica-se a realização desta pesquisa.

Desta forma, o objetivo do trabalho é descrever as características vocais acústicas e de TMF de mulheres adultas com nódulos vocais.

\section{MÉTODO}

Este trabalho foi uma pesquisa exploratória, de campo, transversal, descritiva e quantitativa. $O$ estudo foi desenvolvido com um grupo de 20 indivíduos do sexo feminino, com idades entre 20 e 40 anos. Dez sujeitos com diagnóstico otorrinolaringológico de nódulos vocais formaram o grupo com nódulos vocais (GCN) e os outros dez, com diagnóstico de laringe normal, formaram o grupo sem nódulos vocais (GSN).

Para constituir os grupos pesquisados, os critérios de inclusão foram: ser do sexo feminino, ter idade entre 20 e 40 anos, possuir laudo otorrinolaringológico de nódulos vocais sem outras afecções ou disfunções laríngeas associadas - para o GCN, e laudo otorrinolaringológico de laringe normal para o GSN.

Os critérios de exclusão para o GCN foram: apresentar outras afecções laríngeas além dos nódulos vocais e para o GSN: apresentar afecções laríngeas ou queixas vocais como falhas na voz, sensação de ardência na garganta, fadiga vocal, rouquidão, visto que são variáveis que poderiam interferir nos resultados da pesquisa, pois são sintomas sugestivos de algum tipo de afecção vocal comportamental ou orgânica ${ }^{7,13}$.

Ainda, como critérios de exclusão para ambos os grupos, consideraram-se: ter realizado tratamento otorrinolaringológico e/ou fonoaudiológico prévios, descartando dessa forma a possibilidade de que o sujeito tivesse qualquer alteração vocal (ainda que já tratada) ou certo condicionamento vocal gerado pelo treino com técnicas vocais; apresentar quadro gripal ou alergias respiratórias, pois ambos podem ocasionar edema nas pregas vocais, ou outra doença limitadora da produção vocal durante as avaliações; hábitos de tabagismo e/ou etilismo, já que o tabaco e o álcool são agressivos à laringe e podem gerar alterações vocais orgânicas 7,13; apresentar história de doenças gástricas ${ }^{14}$, psiquiátricas, endocrinológicas, neurológicas ${ }^{7,13}$; alterações hormonais decorrentes de período menstrual e pré-menstrual ou de gravidez, as quais foram investigadas por meio de anamnese aberta ${ }^{15}$; alterações auditivas, pelo fato de poderem provocar alterações no automonitoramento vocal e, consequentemente, comprometerem a qualidade vocal ${ }^{16,17}$; bem como influenciarem a compreensão das ordens no momento das avaliações.

Depois de aplicados os critérios de inclusão e de exclusão e de formados os GCN e GSN, iniciouse a coleta dos dados. Primeiramente, foi realizada a coleta das medidas dos TMF de /a/, /i/, /u/, /s/ e /z/ com cronômetro (Cassio), pedindo aos sujeitos que, em pé e após uma inspiração máxima, sustentassem ao máximo os referidos fonemas por três vezes cada um, em frequência e intensidade habituais, utilizando-se a média dos três valores obtidos. Como valores de normalidade para mulheres adultas jovens, consideraram-se os TMF em torno de 14 a $25 \mathrm{~s}^{8,9,13}$. Abaixo deste valor, foram considerados TMF sugestivos de predomínio aerodinâmico ou escape aéreo à fonação. Acima deste valor, foram considerados TMF sugestivos de predomínio mioelástico ou excesso de tensão glótica.

A relação $s / z$ foi calculada dividindo-se o maior valor do TMF /s/ pelo maior valor do TMF /z/, considerando-se como normalidade a faixa entre 0,8 e 1,2; como predomínio mioelástico laríngeo, valores abaixo de 0,8; e como predomínio aerodinâmico, valores acima de $1,2^{9,13}$. 
Posteriormente, foi realizada a avaliação vocal acústica computadorizada. Esta foi desempenhada em sala tratada acusticamente (material especial sonex) com ruído inferior a $50 \mathrm{~dB}{ }^{13}$. A gravação de voz ocorreu diretamente no computador (Desktop Pentium III $500 \mathrm{mHz}$ ), por meio de um microfone condensador estéreo, omnidirecional, com sensitividade de $35 \mathrm{~dB}$ (Shure, MP66). Foi solicitado ao sujeito que ficasse em pé, com os braços estendidos ao longo do corpo e que realizasse a emissão sustentada da vogal /a/ em freqüência e intensidade habituais. O microfone foi instalado em um pedestal e posicionado a um ângulo de $90^{\circ}$ e a $5 \mathrm{~cm}$ da boca do sujeito durante toda a gravação, a fim de evitar interferências no sinal e manter uma proporção sinal-ruído elevada ${ }^{12,13,18}$.

A análise da onda sonora foi realizada pelo Multi-Dimensional Voice Program Advanced Model 5105 (MDVPA), versão 3.3 do programa Multi Speech da Kay PENTAX, eliminando-se o início e o fim da emissão, por suas características irregulares, utilizando-se um tempo mínimo de 3,5s para a análise ${ }^{18}$.

As medidas acústicas fornecidas pelo programa e que foram analisadas nesta pesquisa foram: frequência fundamental (f0), cociente de perturbação do pitch (PPQ), cociente de perturbação da amplitude (APQ), cociente de variação de frequência fundamental (vfO), índice de fonação suave (SPI), e proporção ruído-harmônico (NHR).

Este estudo foi desenvolvido após a aprovação pelo Comitê de Ética em Pesquisa (CEP) da instituição de origem (074/2005) e a autorização pelos sujeitos da pesquisa, mediante assinatura do termo de consentimento livre e esclarecido (CONEP 196/96).

Realizou-se estatística descritiva comparandose o resultado do GCN com o do GSN em cada uma das medidas vocais do MDVPA e com sua normalidade para o sexo feminino. Para a análise estatística das medidas da média dos TMF das vogais /a, i, u/ e da relação s/z, aplicou-se o teste exato de Fisher, ao nível de significância de 5\%.

\section{RESULTADOS}

A Tabela 1 mostra o resultado das medidas dos TMF das vogais /a/, /i/ e /u/ e a relação s/z classificados em resultados normais, com predomínio aerodinâmico e com predomínio mioelástico, com o resultado do teste exato de Fisher.

$\mathrm{Na}$ Tabela 2, são expostos os resultados da análise acústica das medidas de PPQ, APQ, f0, vf0, NHR, e SPI, extraídos por meio do MDVPA e evidenciando a normalidade proposta pelo programa para o sexo feminino.

Tabela 1 - Resultados das medidas de TMF e relação s/z para o GSN e o GCN e do teste exato de Fisher

\begin{tabular}{|c|c|c|c|c|c|c|c|}
\hline GRUPOS & & $\begin{array}{c}\text { GSN } \\
(n=10)\end{array}$ & & & $\begin{array}{c}\text { GCN } \\
(n=10)\end{array}$ & & Fisher \\
\hline \multirow[b]{2}{*}{ RESULTADOS } & \multirow[b]{2}{*}{$\begin{array}{c}\text { Norma } \\
\text { I (\%) }\end{array}$} & \multicolumn{2}{|c|}{$\begin{array}{c}\text { Alterado } \\
(\%)\end{array}$} & \multirow[b]{2}{*}{$\begin{array}{l}\text { Norma } \\
\text { I (\%) }\end{array}$} & \multicolumn{2}{|c|}{$\begin{array}{c}\text { Alterado } \\
\text { (\%) }\end{array}$} & \multirow{2}{*}{$\begin{array}{c}\text { VALOR-P* } \\
\qquad<0.05\end{array}$} \\
\hline & & $\begin{array}{l}\text { Predom. } \\
\text { Aerod. } \\
(\%)\end{array}$ & $\begin{array}{c}\text { Predom. } \\
\text { Mioel. } \\
(\%) \\
\end{array}$ & & $\begin{array}{c}\text { Predom. } \\
\text { Aerod. } \\
(\%) \\
\end{array}$ & $\begin{array}{c}\text { Predom. } \\
\text { Mioel. } \\
(\%) \\
\end{array}$ & \\
\hline $\begin{array}{c}\text { TMF } \\
/ \mathrm{a} /, / \mathrm{i} /, / \mathrm{u} /\end{array}$ & 90 & 10 & 0 & 70 & 30 & 0 & $=0.582$ \\
\hline $\begin{array}{c}\text { Relação } \\
\text { s/z }\end{array}$ & 100 & 0 & 0 & 70 & 30 & 0 & $=0.211$ \\
\hline
\end{tabular}

Legenda: TMF - tempos máximos de fonação; GSN - grupo sem nódulos; GCN - grupo com nódulos; *VALOR-P - referente ao teste exato de Fisher para comparação entre os grupos 
Tabela 2 - Resultados descritivos das medidas vocais acústicas de fo, PPQ, vf0, APQ, vAm, NHR e SPI para o GCN e o GSN

\begin{tabular}{cccc}
\hline \multirow{2}{*}{ MEDIDAS } & $\begin{array}{c}\text { Valores de normalidade } \\
\text { MDVP - Mulheres }\end{array}$ & GCN & MÉDIA \\
\cline { 3 - 4 } & de 145,22 a $243,97 \mathrm{~Hz}$ & 201,76 & GSN \\
\hline f0 (Hz) & 0,840 & 0,61 & 200,11 \\
PPQ (\%) & 1,149 & 1,12 & 0,66 \\
vfO (\%) & 3,07 & 1,62 & 1,58 \\
APQ (\%) & 10,743 & 8,54 & 10,33 \\
vAm (\%) & 0,190 & 0,11 & 0,13 \\
NHR (\%) & 14,120 & 18,57 & 23,02 \\
SPI (\%) & &
\end{tabular}

Legenda: f0 - frequência fundamental; $\mathrm{PPQ}$ - cociente de perturbação do pitch; Vf0 - cociente de variação de frequência fundamental; vAm - cociente de variação de amplitude; NHR - proporção ruído-harmônico; SPI - índice de fonação suave.

\section{DISCUSSÃO}

Por se tratar de uma lesão de massa da mucosa das pregas vocais, a ocorrência de nódulos tende a afetar as medidas vocais, podendo dificultar o fechamento glótico, alterando os valores de sustentação vocal o que pode culminar em reduzidos TMF. Na presente pesquisa, dentre os resultados do TMF do GCN observou-se que $30 \%$ dos sujeitos apresentaram média reduzida dos TMF das vogais, sugerindo escape de ar transglótico à fonação, concordando com a literatura 1,7,8,9 (Tabela 1). No entanto, quando comparado ao GSN, não houve diferença significativa nos resultados de TMF indo ao encontro dos estudos que não encontraram diferenças entre indivíduos com e sem nódulos nessas medidas $^{8,9}$.

A relação s/z no GSN, indicou adequadas condições de coaptação glótica ${ }^{9}$. Entretanto, na ocorrência de lesões glóticas de massa, como nos casos de nódulos vocais, o som de /z/ tende a ser reduzido, resultando em valores elevados de relação $\mathrm{s} / \mathrm{z}^{1,7,8}$ conforme observado em $30 \%$ dos sujeitos do GCN desta pesquisa, os quais revelaram predomínio aerodinâmico à fonação, sugerindo falta de eficiência glótica, com escape de ar na laringe com a presença da patologia, convergindo com a literatura ${ }^{1,7,8}$ (Tabela 1). Contudo, a diferença de resultados entre os grupos não se revelou significativa nas medidas da relação s/z, convergindo com outro estudo?.

Estes resultados explicam-se pelo fato de que a presença do nódulo determina um impedimento mecânico à vibração da onda mucosa, com redução na amplitude de ondulação, podendo ocorrer falhas no fechamento laríngeo ${ }^{2}$, o que concorda com um estudo ${ }^{5}$ que encontrou, dentre os seus resultados, que todos os pacientes portadores de nódulos vocais apresentavam fenda glótica associada, facilitando o escape aéreo à fonação.

Alguns autores ${ }^{12}$ sugerem que tanto $\mathrm{O}$ jitter (PPQ) quanto o shimmer (APQ) podem ser relacionados, respectivamente, à aspereza e à rouquidão vocais. Conforme a literatura, na presença de nódulos vocais tem-se frequentemente alteração vocal evidente, sendo a rouquidão e a soprosidade perturbações de alta incidência na presença de tal afecção ${ }^{5,6}$, é possível que o aumento dos valores de jitter e shimmer possa estar associado à presença de tais características vocais.

$\mathrm{Na}$ presença de nódulos vocais, é possível o aumento das medidas de jitter e shimmer ${ }^{2,19}$, o que não ocorreu no presente trabalho (Tabela 2) em que essas medidas ficaram dentro da faixa de normalidade do programa MDVPA para o sexo feminino.

Salienta-se que o aumento de percentuais das medidas de shimmer em indivíduos com nódulos vocais, em relação a um grupo de controle, foi observado em pesquisa ${ }^{10}$. Porém, no mesmo trabalho, encontraram-se os valores de jitter similares entre os grupos com nódulos e de controle, corroborando os achados do presente estudo (Tabela 2). Os resultados do presente estudo se assemelham aos de pesquisa ${ }^{20}$ em que não foi observada diferença entre três grupos de sujeitos: sem patologia laríngea, com nódulos vocais e com edema de Reinke, para os parâmetros de jitter e shimmer.

A medida de NHR reflete a avaliação geral de presença de ruído no sinal glótico que se espalha pelas demais freqüências harmônicas 12,18,19,21,22. Com base nesta afirmação, poder-se-ia esperar aumento desta medida no GCN devido à presença de coaptação laríngea insuficiente pela existência de alteração do fechamento glótico, comumente associada a nódulos laríngeos ${ }^{20}$ e pela aperiodicidade de vibração mucosa decorrente da presença da lesão 2,5, como apontado por estudos ${ }^{2,19,22}$ que 
referem que os parâmetros acústicos relacionados ao ruído glótico são maiores em laringes com patologia. Em oposição a tais pesquisas, os resultados deste trabalho evidenciaram que ambos os grupos apresentaram a medida de NHR dentro da normalidade proposta pelo programa MDVPA para o sexo feminino (Tabela 2), concordando com trabalhos ${ }^{17,20}$ que não observaram diferença significativa do NHR ao comparar grupos de sujeitos sem patologia laríngea, com nódulos e com edema de Reinke.

A medida de SPI evidencia a eficiência laríngea, visto que um alto valor desta pode ser resultante de uma fonação mais suave ou de uma coaptação glótica menor ou ineficiente, sendo um indicativo de quão completo é o fechamento das pregas vocais durante a fonação ${ }^{18}$. No presente trabalho, apenas o GSN apresentou a medida elevada, diferentemente do esperado (Tabela 2). No entanto, este resultado não indica necessariamente uma alteração vocal ${ }^{18}$, podendo inclusive estar evidenciando menor tensão glótica à fonação no grupo sem a presença dos nódulos.

A vf0, uma das medidas de jitter ${ }^{18}$, também se apresentou elevada apenas no GSN (Tabela 2), contrariando a literatura que afirma valores elevados de jitter em casos de nódulos vocais ${ }^{2,19}$ ou valores similares entre sujeitos com e sem nódulos ${ }^{20}$. Como a vf0 mede a variabilidade da f0 em toda a amostra avaliada ${ }^{18}$, ela pode estar sinalizando maior dificuldade de coordenação pneumofonoarticulatória ${ }^{23}$ no GSN, o que pode também estar relacionado ao controle respiratório ou às características da camada muco-ciliar que recobre as pregas vocais ${ }^{19}$.

Em relação à f0, sabe-se que representa importante característica vocal, fornecendo indícios anatômicos e funcionais da laringe. Ela quantifica o número de ciclos vibratórios que a mucosa das pregas vocais realiza por segundo, estando relacionada ao estado de comprimento, massa e tensão das pregas vocais ${ }^{2,12,23}$.

No presente trabalho, os valores de f0 apresentaram-se dentro da faixa de freqüência esperada para o sexo feminino, tanto no GCN quanto no GSN, concordando com outro estudo ${ }^{10}$ que encontrou que os valores de frequência fundamental modal mostraram-se similares nos três grupos analisados: de mulheres com qualidade vocal normal, com fenda triangular médio-posterior e com nódulos vocais.

No entanto, há referência na literatura que contraria esse achado ${ }^{2}$, afirmando que, na presença de nódulos vocais, ocorre diminuição da f0 devido ao aumento de massa das pregas vocais.

Dos resultados obtidos e da discussão com a literatura, depreende-se que ainda não há consenso sobre os achados de medidas de TMF e de características vocais acústicas em presença de nódulos vocais.

O presente estudo evidenciou alterações sugestivas de predomínio aerodinâmico nas medidas de TMF e da relação s/z, embora não significativas estatisticamente, e semelhança nos resultados das medidas acústicas da fonte glótica para mulheres jovens com e sem diagnóstico médico de nódulos vocais, quase sempre dentro da normalidade.

Embora as alterações encontradas nas medidas de TMF e da relação s/z não tenham sido significativas, elas pareceram mais sensíveis ao predomínio aerodinâmico esperado na presença dos nódulos vocais do que as medidas acústicas extraídas por meio do MDVPA.

Afirma-se que a análise acústica da voz permite diferenciar vozes normais e patológicas ${ }^{19}$. Porém, devido à falta de padronização dos achados ${ }^{11}$, às diferenças na programação dos vários sistemas de análise do sinal acústico, e aos métodos utilizados para coleta da emissão ${ }^{12}$ e aos ainda escassos estudos envolvendo características acústicas das disfonias, a generalização dos resultados da análise acústica em relação às patologias laríngeas deve ser evitada e mais pesquisas realizadas.

\section{CONCLUSÕES}

Com base nos resultados deste trabalho, pôde-se concluir que as mulheres jovens com diagnóstico médico de nódulos vocais apresentaram medidas de TMF e de relação s/z sem diferenças significativas em relação às mesmas medidas de mulheres sem afecção laríngea.

Em relação às medidas acústicas da fonte glótica, os resultados foram semelhantes e dentro da normalidade para os grupos de mulheres com e sem diagnóstico de nódulos vocais, com exceção da medida do cociente de variação de frequência fundamental (vf0) que foi elevada no grupo sem nódulos, sugerindo incoordenação penumofonoarticulatória.

Nos grupos estudados, as medidas de TMF e da relação $\mathrm{s} / \mathrm{z}$, mesmo sem diferenças significativas, pareceram mais sensíveis à presença do predomínio aerodinâmico dos nódulos vocais do que as medidas acústicas. 


\section{ABSTRACT}

Purpose: to describe maximum phonation time (MPT) and vocal acoustic characteristics for young adult women with vocal fold nodules. Method: collection of MPT of the vowels /a/, /i/ and /u/; s/z ratio and acoustic measures of the glottal source (Multi Dimensional Voice Program Advanced from Kay PENTAX) of 20 female subjects, aged between 20 and 40 year old, being ten with otorhinolaryngologic diagnosis of vocal fold nodules and ten with normal larynx diagnosis. Results: regarding the MPT, the group with normal larynx (GNL) showed $90 \%$ of normal values and $10 \%$ reduced, while the group with nodules (GWN) showed $70 \%$ of normal values and $30 \%$ reduced; the s/z ratio showed results within normal patterns, for the whole GNL, while the GWN showed $70 \%$ of normal results and $30 \%$ increased; both the groups had all the vocal acoustic measures within normality range, except for the GNL which showed increased vf0. Conclusion: young women with vocal nodules showed MPT and $s / z$ ratio measures with no significant differences in relation to the same measures for women with no laryngeal affection. The acoustic measures of glottal source were similar and within normal limits for both groups, except the quotient of variation of fundamental frequency (vf0) measure which was increased in GNL, suggesting pneumophonoarticulatory incoordination. The MPT and s/z ratio measures, even without significant differences, seemed to be more sensitive to the presence of aerodynamic prevalence of vocal nodules than the acoustic measures.

KEYWORDS: Voice; Voice Disorders; Vocal Cords; Health Evaluation

\section{REFERÊNCIAS}

1. Behlau M, Madazio G, Pontes P. Disfonias organofuncionais. In: Behlau M, organizador. Voz: o livro do especialista. vol 1. Rio de Janeiro: Revinter; 2001. p. 295-341.

2. Elhendi W, Santos S, Matul CR, Rossi M, Dios C, Labella T. Nódulos vocales: puesta al día. ORL-DIPS. 2005; 32(3):142-9.

3. Pontes P, Kyrillos L, Behlau M, De Biase N, Pontes A. Vocal nodules and laryngeal morphology. J Voice. 2002; 16(3):408-14.

4. Neves BMJ, G. Neto J, Pontes P. Diferenciação histopatológica e imunoistoquímica das alterações epiteliais no nódulo vocal em relação aos pólipos e ao edema de laringe. Rev Bras Otorrinolaringol. 2004; 70(4):439-48.

5. Braga JN, Oliveira DSF, Atherino CCT, Schott TCA, Silva JC. Nódulos vocais: análise anátomofuncional. Rev CEFAC. 2006; 8(2):223-9.

6. Cielo CA, Conterno G, Carvalho CDM, Finger LS. Disfonias: relação s/z e tipos de voz. Rev CEFAC. 2008; 10(4):536-47.

7. Pinho SMR. Avaliação e tratamento de voz. In: Pinho SMR. Fundamentos em fonoaudiologia: tratando os distúrbios da voz. 2. ed. Rio de Janeiro: Guanabara Koogan; 2003. p. 3-40.

8. Beber BC, Cielo CA, Siqueira MA. Lesões de borda de pregas vocais e tempos máximos de fonação. Rev CEFAC. 2009; 11(1):134-41.
9. Tavares ELM, Martins RHG. Vocal evaluation in teachers with or without symptoms. J Voice. 2007; 21(4):401-14.

10. Gama AC. Análise acústica da voz: estudo comparativo entre mulheres sem queixa vocal e com disfonia [dissertação]. São Paulo (SP): Escola Paulista de Medicina; 1997.

11. Sader RCM, Hanayama EM. Consideração teóricas sobre a abordagem acústica da voz infantil. Rev CEFAC. 2004; 6(3):312-8.

12. Felippe ACN, Grillo MHM, Grechi TH. Normatização de medidas acústicas para vozes normais. Rev Bras Otorrinolaringol. 2006; 72(5):659-64.

13. Behlau M, Madazio G, Feijó D, Pontes P. Avaliação de voz. In: Behlau M, organizador. Voz: o livro do especialista. vol. 1. Rio de Janeiro: Revinter; 2001. p. 85-246.

14. Kelchner LN, Horne J, Lee L, Klaben B, Stemple JC, Adam S, et al. Reliability of speech-language pathologist and otolaryngologist ratings of laryngeal signs of reflux in an asymptomatic population using the reflux finding score. J Voice. 2007; 21(1):92-100. 15. Figueiredo LC, Gonçalves MIR, Pontes A, Pontes P. Estudo do comportamento vocal no ciclo menstrual: avaliação perceptivo-auditiva, acústica e auto-perceptiva. Rev Bras Otorrinolaringol. 2004; 70(3):331-9.

16. Prado AC. Principais características da produção vocal do deficiente auditivo. Rev CEFAC. 2007; 9(3):404-10. 
17. Bolzan GP, Cielo CA, Brum DM. Efeitos do som basal em fendas glóticas. Rev CEFAC. 2008; 10(2):218-25.

18. Barros APB, Carrara-De Angelis E. Análise acústica da voz. In: Dedivitis RA, Barros APB. Métodos de avaliação e diagnóstico de laringe e voz. São Paulo: Lovise; 2002. p. 201-21.

19. Cappellari VM, Cielo CA. Características vocais acústicas de crianças pré-escolares. Rev Bras Otorrinolaringol. 2008; 74(2):265-72.

20. Gama ACC, Behlau, MS. Estudo da constância de medidas acústicas de vogais prolongadas e consecutivas em mulheres sem queixa de voz e em mulheres com disfonia. Rev Soc Bras Fonoaudiol. 2009; 14(1):8-14.

21. Hamdan AL, Medawar W, Youness A, Bikhazi $\mathrm{H}$, Fuleihan $\mathrm{N}$. The effect of hemodialysis on voice: an acoustic analisys. J Voice. 2005; 19(2):290-5.

22. González BS, Batalla FN, Santos PC, Suarez Nieto C. Índice de incapacidad vocal: factores predictivos. Acta Otorrinolaringol Esp. 2006; 57:101-8.

23. Roman-Niehues G, Cielo CA. Modificações vocais acústicas produzidas pelo som hiperagudo. Rev CEFAC, 2009. no.ahead

doi: 10.1590/S1516-18462011005000002

RECEBIDO EM: 20/08/2009

ACEITO EM: 10/11/2010

Endereço para correspondência:

Shanna Lara Miglioranzi

Av. Presidente Vargas, 1697/06

Santa Maria - RS

CEP: 97015-511

E-mail: shannalara@yahoo.com.br 